\title{
Microbiome-metabolomics analysis of the effects of decreasing dietary crude protein content on goat rumen mictobiota and metabolites
}

\author{
Wen Zhu ${ }^{1}$, Tianwei Liu ${ }^{1}$, Jian Deng ${ }^{1}$, Cong Cong Wei ${ }^{1}$, Zi Jun Zhang ${ }^{1}$, \\ Di Ming Wang ${ }^{2}$, and Xing Yong Chen ${ }^{1}$ *
}

\author{
* Corresponding Author: Xing Yong Chen \\ Tel: +86-055165786328, \\ Fax: +86-055165786328, \\ E-mail: chenxingyong@ahau.edu.cn \\ ${ }^{1}$ College of Animal Science and Technology, \\ Anhui Agricultural University, Hefei 230036, \\ China \\ ${ }^{2}$ Key Laboratory of Molecular Animal \\ Nutirtion, Ministry of Education, Zhejiang \\ University, Hangzhou 310058, China \\ ORCID \\ Wen Zhu \\ https://orcid.org/0000-0002-7118-2791 \\ Tianwei Liu \\ https://orcid.org/0000-0002-2639-1623 \\ Jian Deng \\ https://orcid.org/0000-0002-0190-615X \\ Cong Cong Wei \\ https://orcid.org/0000-0002-2095-3373 \\ Zi Jun Zhang \\ https://orcid.org/0000-0002-9869-7381 \\ Di Ming Wang \\ https://orcid.org/0000-0002-1451-6863 \\ Xing Yong Chen \\ https://orcid.org/0000-0003-3855-0473
}

Submitted Sept 7, 2021; Revised Jan 4, 2022; Accepted Feb 18, 2022

\begin{abstract}
Objective: The objective of this study was to investigate the effects of decreasing dietary crude protein content on rumen fermentation, mictobiota, and metabolites in goats.

Methods: In an 84-day feeding trial, a total of twelve male Anhui white goat kids with initial body weight $15.9 \pm 1.13 \mathrm{~kg}$ were selected and randomly classified into two groups, feeding a normal crude protein diet $(14.8 \% \mathrm{CP}, \mathrm{NCP})$ or a low crude protein diet (12.0\% CP, LCP). At the end of the experimental trial (on day 84), six animals were randomly selected from each group and were slaughtered to collect rumen fluid samples for the analysis of rumen fermentation parameters, microbiome, and metabolome.

Results: The concentrations of ammonia-nitrogen, total volatile fatty acid, acetate, and propionate were decreased $(\mathrm{p}<0.05)$ in the LCP group in comparison with those in the NCP group. The abundances of genera Prevotella, Campylobacter, Synergistetes, and TG5, which were associated with nitrogen metabolism, were lower $(\mathrm{p}<0.05)$ in the LCP group compared with those in the NCP group. The levels of 78 metabolites ( 74 decreased, 4 increased) in the rumen fluid were altered $(\mathrm{p}<0.05)$ by the treatment. Most of the ruminal metabolites that showed decreased levels in the LCP group were substrates for microbial protein synthesis. Metabolic pathway analysis showed that vitamin B6 metabolism was significantly different $(\mathrm{p}<0.05)$ in rumen fluid between the two treatments.

Conclusion: Decreased dietary protein level inhibited rumen fermentation through microbiome and metabolome shifts in goat kids. These results enhance our understanding of ruminal bacteria and metabolites of goat fed a low protein diet.
\end{abstract}

Keywords: Goat; Low Protein; Metabolomic; Microbiome; Rumen

\section{INTRODUCTION}

The nitrogen $(\mathrm{N})$ efficiency (the sum of $\mathrm{N}$ retained in milk and gain divided by $\mathrm{N}$ intake) of ruminants is very low (less than $30 \%$ ), the rest is excreted into urine and feces [1]. The application of a low protein diet has been demonstrated to be an effective way to reduce urinary $\mathrm{N}$ loss and improve $\mathrm{N}$ utilization efficiency in ruminants [2]. However, the decreased microbial protein synthesis and nutrient digestion as a result of reduced dietary protein intake may have negative effects on goat production [3,4]. Thus, to facilitate application of a low protein diet without causing detrimental effects on production, a better understanding of the alterations that occur in response to decreasing protein content diet is needed.

The rumen is a complex microbial ecosystem that harbors a wide variety of microorganisms, including bacteria, archaea, fungi, and protozoa [5]. The ruminal microbiota degrades and ferments dietary compounds into absorbable molecules, including volatile 
fatty acids (VFAs) and amino acids, which can be used by the host animal [6]. Rumen microbiota is regulated by dietary nutritional factors [7]. The protein content is one of the most important dietary nutritional factors affecting microbial growth [8]. For example, Gao et al [9] reported that the relative abundances of Ruminobacter amylophilus and Ruminococcus albus in the rumen of steer were increased with a decreasing dietary protein content. Furthermore, the rumen microbiota also coproduces a wide range of metabolites that play key roles in shuttling information between the microbial symbionts and their host [10]. Thus, a comprehensive analysis of the rumen microbe profile and metabolic processes will offer important insights for understanding the potential limitations of the application of low protein diet.

In our previous study, we found that the average daily gain and feed efficiency were decreased with a reduction in the dietary protein content [4]. We hypothesized that the phenotype differences in growing goats may be due to alterations of the rumen microbiota and metabolism induced by low dietary protein content. Therefore, this study aimed to investigate the effect of the dietary protein reduction on rumen fermentation parameters, rumen microbiota, and metabolism in growing goats.

\section{MATERIALS AND METHODS}

\section{Animals and experimental design}

A detailed description of the experimental design and animal treatment has been reported [4]. Briefly, animal usage was approved by the Animal Care Advisory Committee of the Anhui Agriculture University (SYXK (Wan) 2016-007). Twenty-four healthy male Anhui white goat kids (initial live weight, $15.9 \pm 1.13 \mathrm{~kg}$; age, $109 \pm 4$ days; $\mathrm{n}=24$ ) were randomly distributed into two dietary treatments. Diets differed in crude protein (CP) content as follows: $\mathrm{i} \neg) 148 \mathrm{~g} \mathrm{CP} / \mathrm{kg}$ of dry matter (DM) (normal CP diet, NCP); ii) $120 \mathrm{~g} \mathrm{CP} / \mathrm{kg}$ of DM (low CP diet; LCP). The control diet was formulated to meet the nutrient requirements of $15 \mathrm{~kg}$ meat goat aimed to 100 to $150 \mathrm{~g} / \mathrm{d}$ daily gain according to NRC guidelines [11] (Table 1). The feeding trial was conducted for 14 weeks, with the first 2 weeks used as an adaption period. The animals were fed twice daily at 06:30 and 18:30 h, allowing for approximately $10 \%$ feed refusal, with free access to clean water.

\section{Sample collection and measurement}

At the end of the experiment, six goats were randomly selected from each group and were slaughtered in a local slaughterhouse before morning feeding. Homogenized rumen digesta from each goat was collected and was immediately squeezed through 4 layers of cheesecloth. The $\mathrm{pH}$ of the rumen fluid was measured immediately by a mobile $\mathrm{pH}$ meter (Seven2Go Pro S8; Mettler Toledo Co. Ltd., Shanghai, China).
Table 1. Ingredients and chemical composition of the experimental $\operatorname{diets}^{1)}$

\begin{tabular}{|c|c|c|}
\hline \multirow{2}{*}{ Item } & \multicolumn{2}{|c|}{ Dietary treatments, \% dry matter } \\
\hline & NCP & LCP \\
\hline \multicolumn{3}{|l|}{ Ingredient } \\
\hline Ground corn grain & 13.0 & 21.0 \\
\hline Soybean meal ${ }^{2)}, 43.5 \%$ CP & 24.0 & 16.0 \\
\hline Wheat bran & 7.5 & 7.5 \\
\hline Sodium bicarbonate & 1.0 & 1.0 \\
\hline Salt & 1.0 & 1.0 \\
\hline Dicalcium phosphate & 0.5 & 0.5 \\
\hline Calcium carbonate & 1.0 & 1.0 \\
\hline Premix ${ }^{3)}$ & 1.0 & 1.0 \\
\hline Peanut vine & 28.0 & 28.0 \\
\hline Chinese wild rye & 22.0 & 22.0 \\
\hline \multicolumn{3}{|l|}{ Chemical composition } \\
\hline Organic matter & 86.3 & 88.3 \\
\hline Crude protein & 14.8 & 12.0 \\
\hline Neutral detergent fiber & 46.4 & 44.8 \\
\hline Acid detergent fiber & 31.8 & 31.2 \\
\hline Ether extract & 3.74 & 3.27 \\
\hline Digestible energy ${ }^{4}(\mathrm{MJ} / \mathrm{kg})$ & 13.9 & 13.8 \\
\hline \multicolumn{3}{|c|}{$\begin{array}{l}\text { NCP, normal crude protein diet; LCP, low crude protein diet. } \\
\text { 1) Cited from Zhu et al [4]. } \\
\text { 2) Soybean meal contained: } 89.5 \% \text { dry matter, } 43.5 \% \text { crude protein, } 28.2 \% \\
\text { neutral detergent fiber and } 10.5 \% \text { acid detergent fiber. } \\
\text { 3) Formulated to provide (per } \mathrm{kg} \text { of dry matter): } 600,000 \text { IU of vitamin } \mathrm{A} \text {, } \\
80,000 \mathrm{IU} \text { of vitamin } \mathrm{D}_{3}, 5,000 \mathrm{IU} \text { of vitamin } \mathrm{E}, 8,000 \mathrm{mg} \text { of } \mathrm{Zn}, 60 \mathrm{mg} \text { of } \\
\text { Se, } 200 \mathrm{mg} \text { of I, } 9,400 \mathrm{mg} \text { of Fe, } 72 \mathrm{mg} \text { of } \mathrm{Co}, 10,400 \mathrm{mg} \text { of } \mathrm{Mn} \text {, and } 1,600 \\
\text { mg of Cu; Peanut vine contained: } 91.3 \% \text { dry matter, } 7.17 \% \text { crude protein, } \\
57.2 \% \text { neutral detergent fiber, and } 50.5 \% \text { acid detergent fiber on dry } \\
\text { matter basis; Chinese wild rye contained: } 89.6 \% \text { dry matter, } 6.7 \% \text { crude } \\
\text { protein, } 67.5 \% \text { neutral detergent fiber, and } 38.2 \% \text { acid detergent fiber on } \\
\text { dry matter basis; } \\
\text { 4) Calculated according to nutrient requirements of small ruminants. }\end{array}$} \\
\hline
\end{tabular}

One subsample of each rumen filtrate sample was acidified by $25 \% \mathrm{HPO}_{3}(4: 1 ; \mathrm{v}: \mathrm{v})$ for analysis of VFAs, as described by $\mathrm{Hu}$ et al [12]. Another 1.0-mL subsample was used to determine the ammonia-N content by the colorimetric method, in accordance with the method reported by Chaney and Marbach [13]. Two other 5.0-mL subsamples were infused into two $10-\mathrm{mL}$ spiral centrifuge tubes for analysis of microbiota and metabolites. All collected rumen fluid samples were snap-frozen in liquid nitrogen immediately, transferred to the laboratory, and stored at $-80^{\circ} \mathrm{C}$ for further analysis.

\section{Rumen microbial 16S rDNA sequencing}

Rumen microbial genomic DNA was extracted using the QIAamp DNA stool mini kit (QIAGEN, Inc., Venlo, Netherlands). The integrity and concentration of the extracted DNA was evaluated using agarose gel ( $1 \%$, wt/vol) electrophoresis and a NanoDrop spectrophotometer (Thermo Fisher Scientific, Waltham, MA, USA), respectively.

The amplicons of the V3-V4 regions of the 16S rRNA gene were prepared using the primers 520F/802R with dual- 
index 7-bp barcodes according to the method described by Wang et al [14]. After purification and quantification, amplicons for all samples were pooled in equimolar concentrations and sequenced on an Illumina MiSeq platform at Shanghai Personal Biotechnology Co., Ltd (Shanghai, China). Raw sequence data in this study are available from the National Center for Biotechnology Information Sequence Read Archive database under accession numbers SRR12597087- SRR 12597110 .

\section{Sequence processing and statistical analyses}

Raw sequences with a quality score $<20$, length $<150 \mathrm{bp}$, and mononucleotide repeats $>8$ bp were filtered by QIIME pipeline (v1.8.0). Paired-end reads were merged using FLASH (1.2.8). Operational taxonomic units (OTUs) were clustered at $97 \%$ sequence similarity by UCLUST, then were classified by BLAST against the Greengenes Database (version 13.8). For alpha diversity analysis, the rarefaction and rank curves were plotted, and the Chaol index, abundance-based coverage estimator (ACE) metric, Shannon diversity index, and Simpson index were implemented in QIIME. The beta-diversity analysis was visualized using principal-coordinate-analysis (PCoA) plots, which was estimated based on unweighted and weight UniFrac distance metrics. The analysis of similarities (ANOSIM) and permutational multivariate analysis of variance (ADONIS) were used to calculate the differences between the two groups.

\section{Rumen fluid metabolomics processing}

Rumen samples were prepared according to the procedures described by Sun et al [15]. A total of $10 \mu \mathrm{L}$ of each sample was mixed together as the quality control sample. A UHPLC system (1290; Agilent Technologies, Santa Clara, CA, USA) equipped with an UPLC BEH Amide column $(1.7 \mu \mathrm{m} \times 2.1$ $\mathrm{mm} \times 100 \mathrm{~mm}$; Waters, Milford, MA, USA) coupled to TOF 6600 (Q-TOF; AB Sciex, Framingham, MA, USA) was used for metabolic profiling analyses. A binary solvent system consisting of $25 \mathrm{mM} \mathrm{NH}_{4} \mathrm{Ac}$ and $25 \mathrm{mM} \mathrm{NH}_{4} \mathrm{OH}$ in water ( $\mathrm{pH}$ 9.75) (solution A) and acetonitrile (solution B) was used with the following elution gradient procedure: $0.5 \mathrm{~min}$ (95\% B); $6.5 \mathrm{~min}$ (95\% to $65 \% \mathrm{~B}) ; 1 \mathrm{~min}(65 \%$ to $40 \% \mathrm{~B}) ; 1 \mathrm{~min}$ (40\% B); $0.1 \mathrm{~min}$ (40\% to $95 \% \mathrm{~B}$ ); and $2.9 \min (95 \% \mathrm{~B})$. The column temperature and auto-sampler temperature were $25^{\circ} \mathrm{C}$ and $4^{\circ} \mathrm{C}$, respectively. The flow rate was $0.50 \mathrm{~mL} / \mathrm{min}$, and the injection volume was $2 \mu \mathrm{L}$ for the positive or negative mode.

The TripleTOF 6600 mass spectrometer (AB Sciex, USA) was used to obtain mass spectrometer (MS)/MS spectra with information-dependent acquisition. In each cycle, the most intensive 12 precursor ions with intensity greater than 100 were chosen for fragmentation. The collision energy (CE) was $30 \mathrm{eV}$. The cycle time was $0.56 \mathrm{~s}$. The parameters of the electronic spray ionization source were as follows: $60 \mathrm{psi}$ for gas 1,60 psi for gas 2,35 psi for the curtain gas, $600^{\circ} \mathrm{C}$ source temperature, $5,000 \mathrm{~V}$ or $-4,000$ ion spray voltage floating in positive or negative modes, respectively.

\section{Metabolomics data analysis}

The MS raw data were processed by R package XCMS (version 3.22), which yielded a data matrix containing the retention time (RT), mass-to charge ratio $(\mathrm{m} / \mathrm{z})$ values, and peak intensity. Minfrac and cut-off were set as 0.5 and 0.3 , respectively. The metabolites were identified using an in-house MS2 database (Biotree Biotech. Co., Ltd, Shanghai, China). A total of 3,740 resulting peaks were imported to the SIMCA software package (V15.0.2; Sartorius Stedim Data Analytics AB, Umea, Sweden) for principal component analysis (PCA) and orthogonal partial least-squares discriminant analysis (OPLS-DA). Additionally, a 7-fold permutation test with 200 random permutations in the OPLS-DA model was applied to verify the validity and robustness of the model. The metabolites were identified and confirmed by the online Kyoto encyclopedia of genes and genomes (https://www. kegg.jp/kegg/pathway.html). MetaboAnalyst 4.0 (https:// www.metaboanalyst.ca/) was utilized for pathway topology analysis.

\section{Statistical analysis}

The rumen fermentation characteristics and metabolite data were analyzed using SPSS (version 23.0; SPSS Inc., Chicago, IL, USA). One-way analysis of variance was performed to analyze the differences in $\mathrm{pH}$, ammonia- $\mathrm{N}$, and VFAs between the two groups. A significant change was observed at $\mathrm{p}<0.05$.

The Metastats test in Mothur (version 1.30.1) was used to identify rumen bacteria that showed different abundances between the groups. Significance was observed at $\mathrm{p}<0.05$ and a risk of false discovery ( $q$ value) $<0.10$. The value of variable importance in projection (VIP) was obtained from the OPLS-DA model, and variables with VIP $>1$ and $p<0.05$ (student's t-test) were considered to indicate significant differences in the levels of metabolites.

\section{RESULTS}

\section{Rumen fermentation parameters}

Rumen $\mathrm{pH}$ was not significantly different $(\mathrm{p}>0.05)$ between the LCP and the NCP groups (Table 2). The rumen fluid concentrations of ammonia- $\mathrm{N}$, total VFA, acetate, and propionate were significantly lower $(\mathrm{p}<0.05)$ in the LCP group in comparison with those in the NCP group. The concentrations of butyrate, valerate, and acetate to propionate ratio were not significantly altered by changes in the dietary $C P$ level $(\mathrm{p}>0.05)$. 
Table 2. Effects of low crude protein diet on rumen fermentation characteristics in fattening goats $(n=6)$

\begin{tabular}{|c|c|c|c|c|}
\hline \multirow{2}{*}{ Items } & \multicolumn{2}{|c|}{ Treatment } & \multirow{2}{*}{ SEM } & \multirow{2}{*}{ p-value } \\
\hline & NCP & LCP & & \\
\hline $\mathrm{pH}$ & 6.64 & 6.51 & 0.038 & 0.49 \\
\hline Ammonia-nitrogen (mg/dL) & $10.5^{\mathrm{a}}$ & $8.94^{b}$ & 0.431 & 0.03 \\
\hline Total volatile fatty acid (mM) & $74.3^{\mathrm{a}}$ & $65.1^{b}$ & 2.51 & 0.03 \\
\hline Acetate $(\mathrm{mM})$ & $52.6^{a}$ & $45.8^{b}$ & 1.71 & 0.04 \\
\hline Propionate (mM) & $13.1^{\mathrm{a}}$ & $11.2^{\mathrm{b}}$ & 0.46 & 0.02 \\
\hline Butyrate $(\mathrm{mM})$ & 7.78 & 7.28 & 0.37 & 0.59 \\
\hline Valerate (mM) & 0.81 & 0.83 & 0.029 & 0.67 \\
\hline Acetate:propionate & 3.73 & 3.75 & 0.121 & 0.68 \\
\hline
\end{tabular}

NCP, normal crude protein diet; LCP, low crude protein diet; SEM, standard error mean.

a,b Values within a row with different superscripts differ significantly at $p<0.05$. The $p$-values were determined using one-way analysis of variance.

\section{Rumen fluid microbial communities}

After quality filtering, a total of 388,089 high-quality sequences were obtained with an average of 32,341 \pm 58 (mean \pm standard deviation [SD]) sequences per sample, and 1,618 \pm 299 (mean \pm SD) OTUs were observed based on $97 \%$ similarity. Good's coverage for all rumen fluid samples were greater than $99.5 \%$. The flattened rarefaction (Supplementary Figure S1A) and rank abundance curves (Supplementary Figure S1B) demonstrated that most of the rumen microbes in the samples were quantified, and the data could be used for further analysis. Alpha diversity analysis indicated that neither the richness estimators (Chao 1 and ACE value) nor the diversity indices (Shannon and Simpson indices) were affected $(\mathrm{p}>0.05)$ by
Table 3. Alpha diversity of rumen bacterial communities in fattening goats fed the low crude protein diet (LCP) and the normal crude protein diet (NCP)

\begin{tabular}{lcccc}
\hline \multirow{2}{*}{ Items } & \multicolumn{2}{c}{ Treatment } & \multirow{2}{*}{ SEM } & p-value \\
\cline { 2 - 3 } & NCP & LCP & & \\
\hline Good's coverage & 0.995 & 0.995 & 0.0069 & 0.99 \\
Chao1 value & 1,594 & 1,677 & 50.1 & 0.43 \\
ACE value & 1,607 & 1,665 & 48.9 & 0.57 \\
Shannon indices & 8.59 & 8.62 & 0.127 & 0.91 \\
Simpson indices & 0.991 & 0.992 & 0.0161 & 0.84 \\
\hline
\end{tabular}

NCP, normal crude protein diet; LCP, low crude protein diet; SEM, standard error mean; ACE, abundance-based coverage estimator.

The $p$-values were determined using student's t test.

the LCP (Table 3).

Weighted Unifrac distances did not show complete separation between the two groups (Figure 1B). However, the PCoA based on the unweighted UniFrac distances indicated rumen fluid samples from the LCP and NCP groups clustered differently (ANOSIM, $\mathrm{p}<0.05$; ADONIS, $\mathrm{p}<0.05$ ) (Figure $1 \mathrm{~A})$.

The rumen-microbiota distributions at the phylum and genus levels are shown in Figure 2. Among the identified phyla, Firmicutes was the most dominant, with an average abundance of $62.3 \% \pm 13.9 \%$ (mean $\pm \mathrm{SD})$, followed by Bacteroidetes $(20.2 \% \pm 16.2 \%)$, Tenericutes $(3.85 \% \pm 1.49 \%)$, Verrucomicrobia $(3.20 \% \pm 1.00 \%)$, and Proteobacteria $(3.15 \%$ $\pm 1.54 \%$ ) (Figure 2A). At the genus level, Succiniclasticum $(4.06 \% \pm 1.81 \%)$, Prevotella $(3.00 \% \pm 1.32 \%)$, Butyrivibrio (2.53\% $\pm 0.91 \%)$, TG5 (1.99\% $\pm 1.30 \%)$, and Treponema $(1.73 \%$
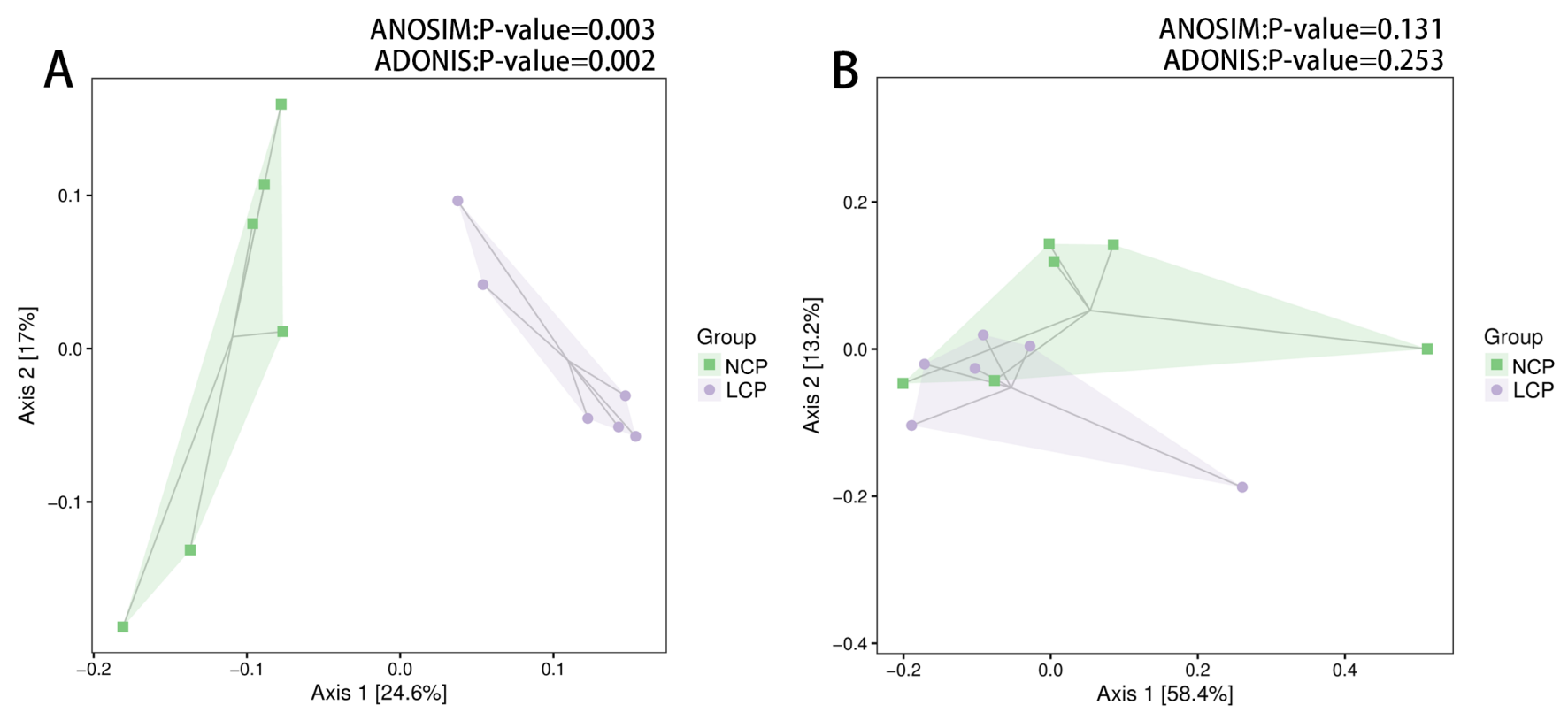

Figure 1. Principal coordinate analysis of unweighted (A) and weight (B) UniFrac distances of all 12 rumen samples. NCP, normal crude protein diet; LCP, low crude protein diet. ANOSIM, analysis of similarities; ADONIS, permutational multivariate analysis of variance. 
A

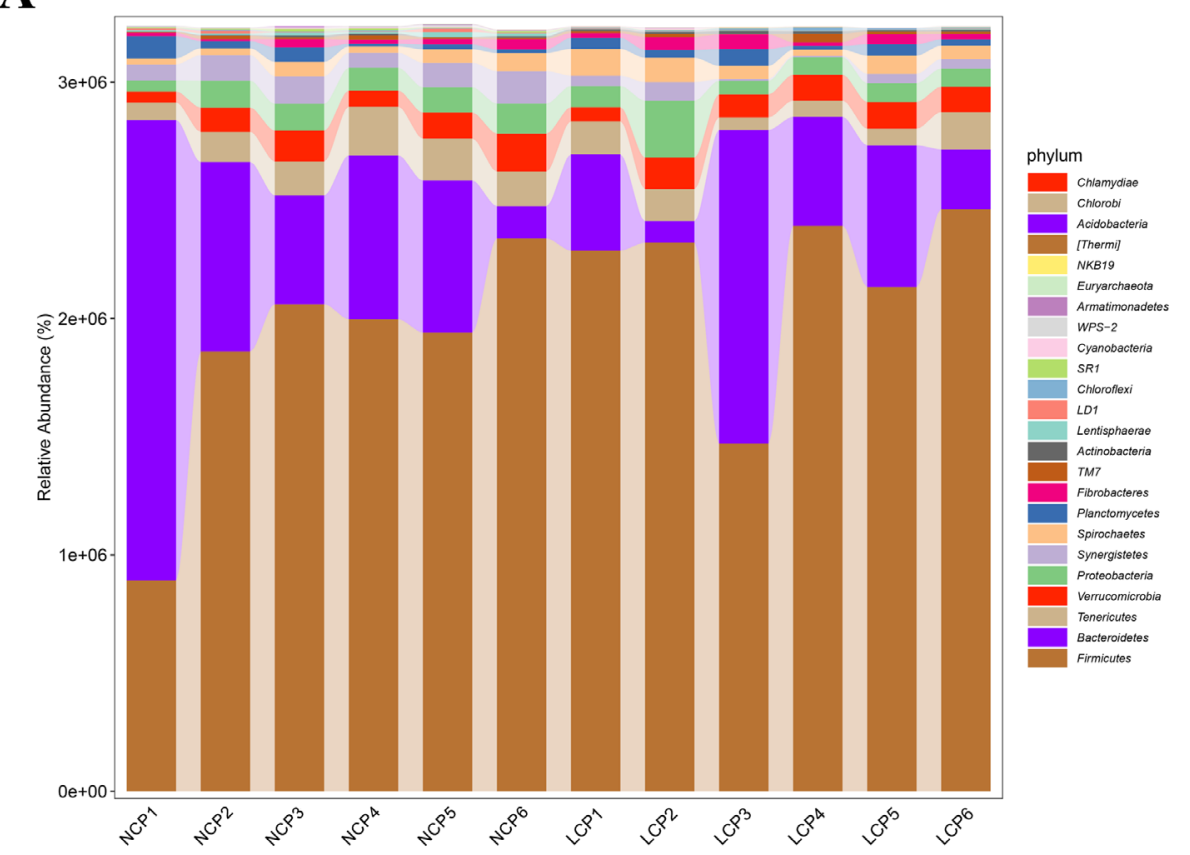

B

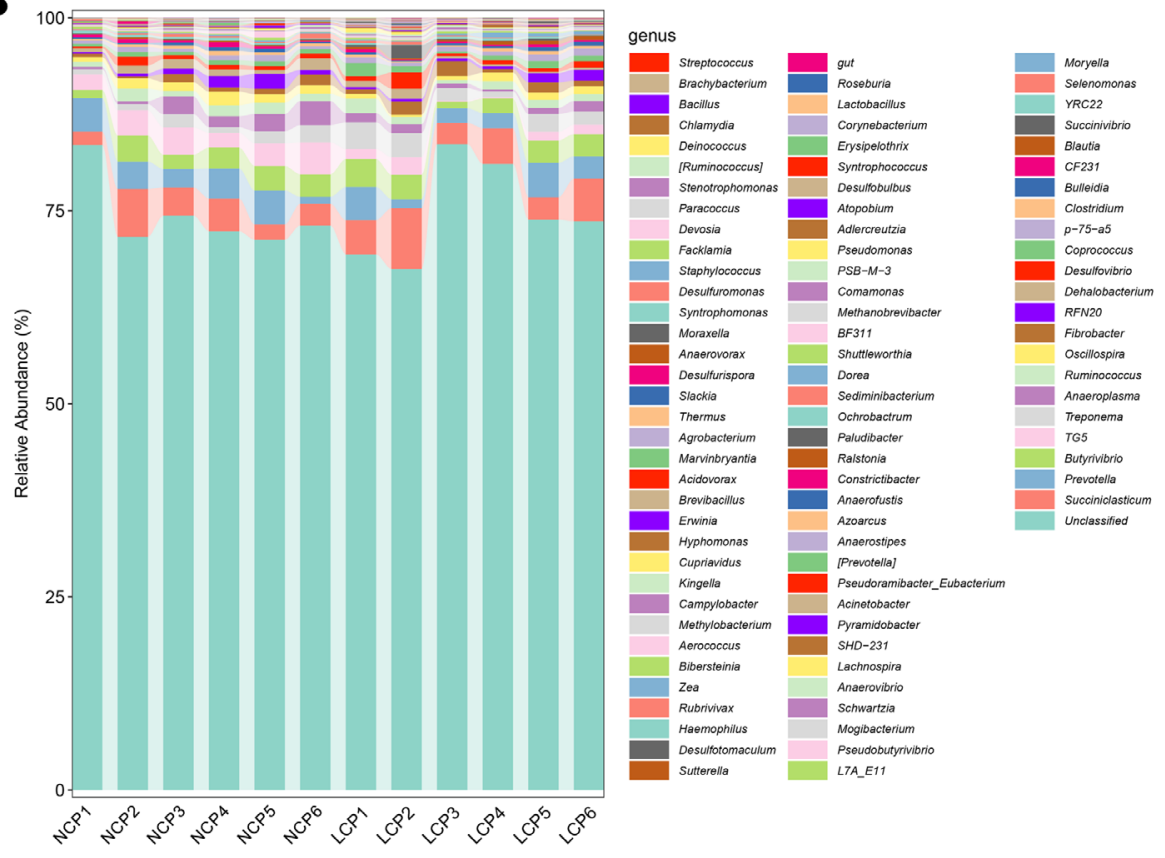

Figure 2. The relative abundance of rumen microbiota at the phylum (A) and genus level (B). NCP, normal crude protein diet; LCP, low crude protein diet.

$\pm 0.93 \%$ ) were considered as high-abundance (Figure 2B).

The bacteria that were significantly different between the two groups at the phylum and genus levels are shown in Figure 3. At the phylum level (Figure 3A), in comparison with the NCP group, the LCP group showed lower $(\mathrm{p}<0.05)$ relative abundance of Synergistetes, Lentisphaerae, LD1, SR1, WPS-2, whereas Actinobacteria showed higher relative abundances $(\mathrm{p}<0.05)$. At the genus level (Figure $3 \mathrm{~B})$, the LCP group showed lower $(\mathrm{p}<0.05)$ relative abundances of Prevotella, TG5, and Campylobacter and higher $(\mathrm{p}<0.05)$ relative abundances of Blautia, Azoarcus, PSB-M-3, and Roseburia.

\section{Rumen fluid metabolic profiles}

Based on the analysis of untargeted metabolomics data, PCA plots were not completely separated between the LCP and NCP groups (Figure 4A, B). However, the analysis of OPLS- 
A

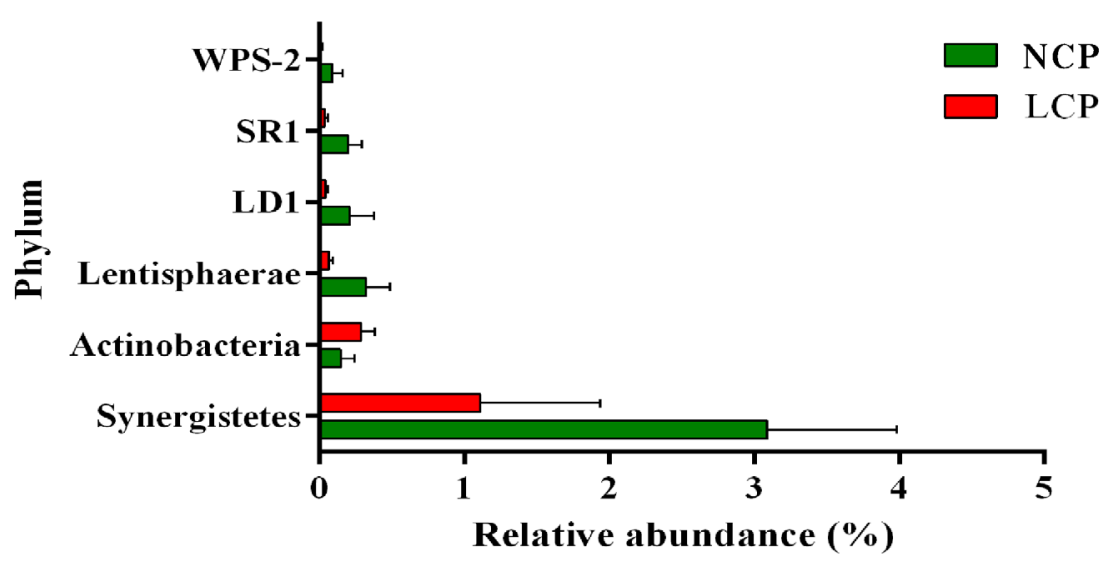

B

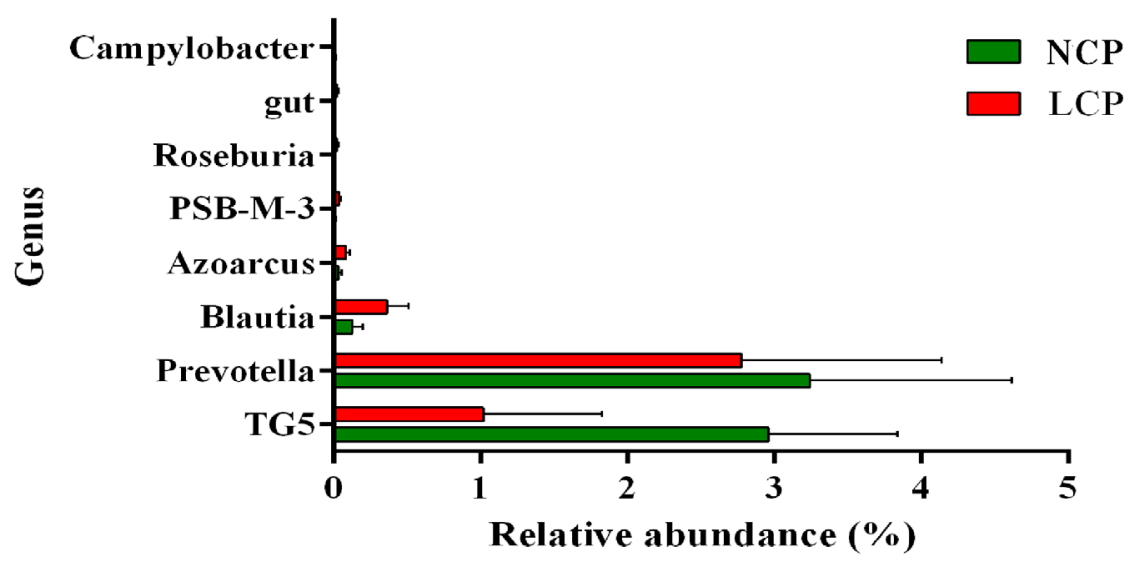

Figure 3. Different composition of rumen microbiota at the phylum (A) and genus (B) levels between the low crude protein diet (LCP) and the normal crude protein diet (NCP) (Metastats test).

$\mathrm{DA}\left(\mathrm{R}^{2} \mathrm{Y}=0.877, \mathrm{Q}^{2}=0.437\right.$ for the positive ion mode; $\mathrm{R}^{2} \mathrm{Y}$ $=0.859, \mathrm{Q}^{2}=0.455$ for the negative ion mode) of the metabolic profiles showed a noticeable separated cluster between the LCP and NCP groups (Figure 4C, D). The corresponding $\mathrm{Q}^{2}$ values of the OPLS-DA model are negative $(-0.58$ and -0.62 in positive and negative ion mode, respectively) (Figure $4 \mathrm{E}, \mathrm{F}$ ), indicated the valid and at low risk of over fitting of the statistical models. In addition, in PCA and OPLSDA plots we found all samples of the two groups within the 95\% Hotelling's T-Squared Ellipse.

The levels of 78 metabolites in the rumen fluid differed significantly $(\mathrm{p}<0.05)$ between the LCP and NCP groups $(p<0.05$ and VIP $>1)(47$ in the positive-ion mode and 31 in the negative-ion mode). Among these 78 metabolites, the levels of 74 were lower and 4 were higher in the LCP group in comparison with the NCP group (Supplementary Table S1). Based on the classification information, the differential metabolites were mainly classified into lipids and lipid-like molecules, phenylpropanoids and polyketides, organoheterocyclic compounds, organic oxygen compounds, benzenoids, alkaloids and derivatives, and organic acids and derivatives.
Data from the negative and positive modes were combined. The significantly altered metabolites between the LCP and NCP groups were enriched in pathways of vitamin $B_{6}$ metabolism, glycerolipid metabolism, pantothenate and CoA biosynthesis, beta-Alanine metabolism, and pyrimidine metabolism (Figure 5). The detailed results of the pathway analyses are shown in Table 4 . The pathway of vitamin $\mathrm{B}_{6}$ metabolism was significantly altered $(\mathrm{p}<0.05)$ by the low protein diet.

\section{DISCUSSION}

Dietary CP level is a vital factor in the growth of ruminants. Decreasing CP level decreased the CP intake and growth performance of goat kids [4]. Ammonia-N is the final product of the feed protein decomposition, and a positive correlation between $\mathrm{CP}$ intake and ammonia- $\mathrm{N}$ has been reported by Xia et al [16]. In the present study, low protein diet decreased ammonia- $\mathrm{N}$ concentration, which is consistent with the previous study [17]. The VFAs, which are the primary energy source for ruminants, are the main fermentation product in 

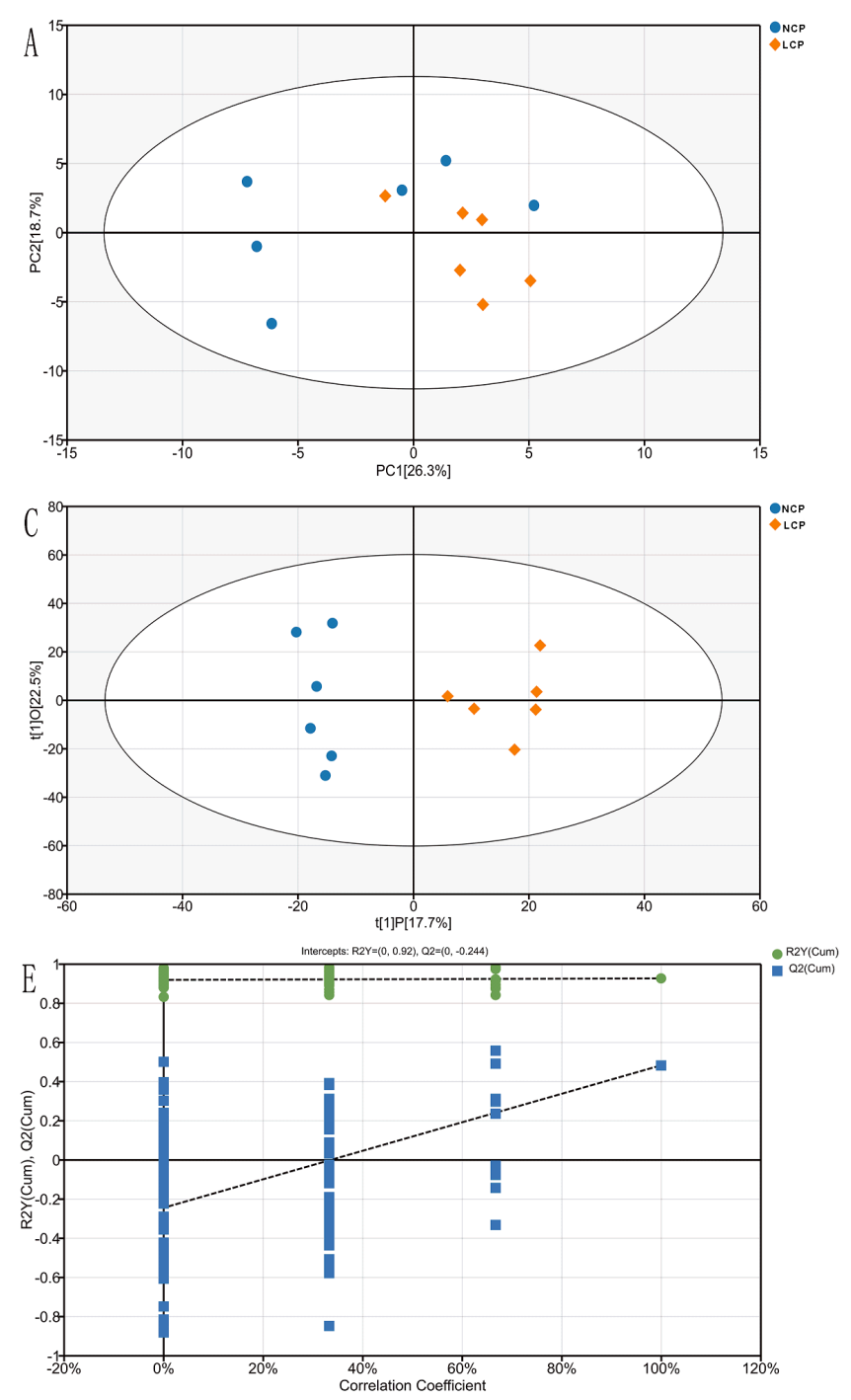
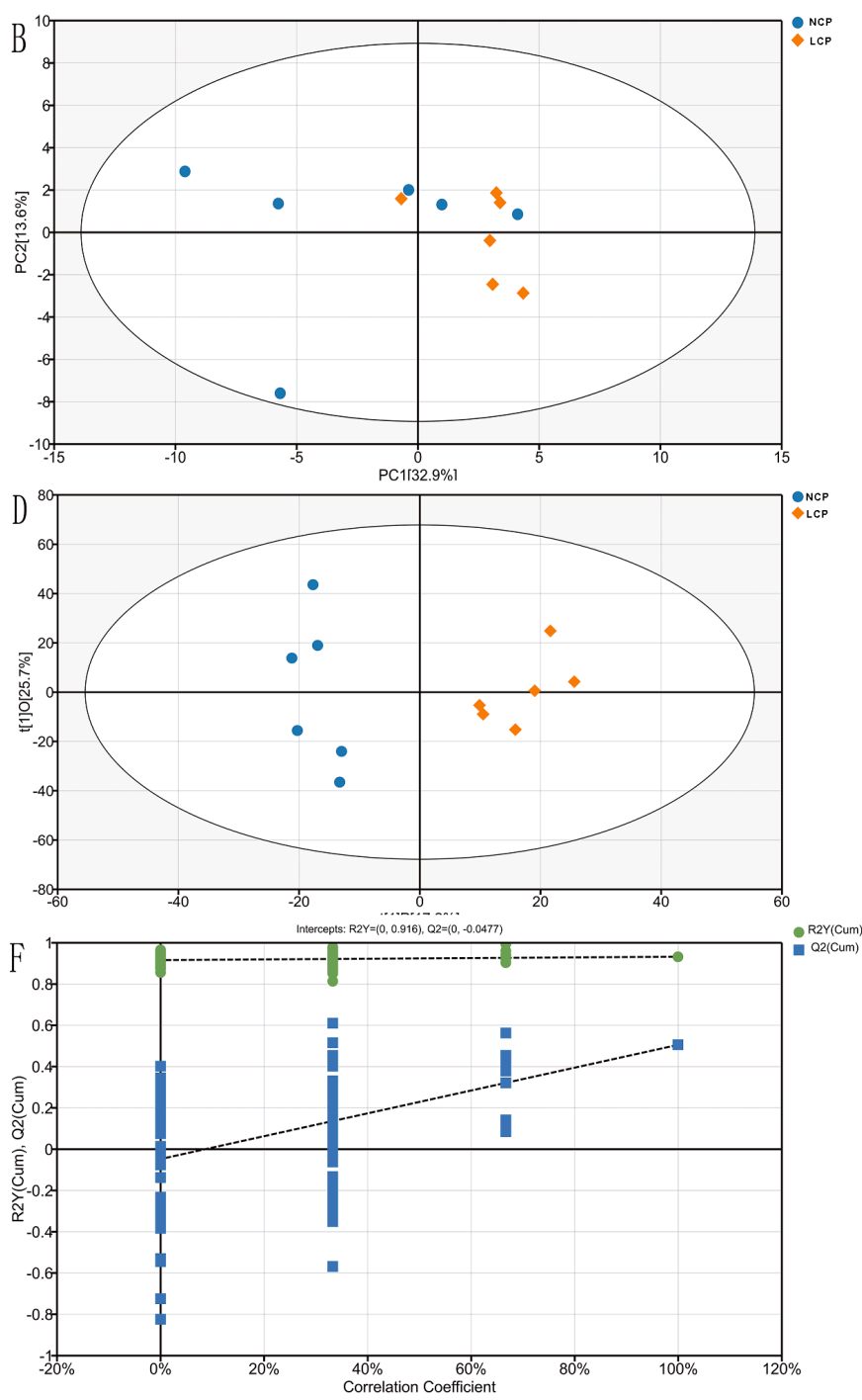

Figure 4. Identification of discriminating biomarkers by metabolomics analysis between the low crude protein diet (LCP) and the normal crude protein diet (NCP). The principal component analysis (A) and orthogonal partial least-squares discriminant analysis (C) score plot are in negative ion mode. The principal component analysis (B) and orthogonal partial least-squares discriminant analysis (D) score plot are in positive ion mode. Permutation tests conducted with 200 random permutations in the orthogonal partial least-squares discriminant analysis model is built for negative (E) and positive ion mode (F).

the rumen [18]. The decreased concentration of total VFA, acetate, and propionate with LCP indicated less energy supply to goats, which provided a potential explanation for the decreased growth performance as was observed in our previous study [4].

Rumen microbiota composition shifts in response to dietary CP level has been reported in previous study [16]. In the current study, specific microbes were observed to differ between the two groups. At the genus level, the relative abundances of Prevotella, TG5, and Campylobacter were lower in the LCP group than in the NCP group. Prevotella has been reported to be involved in the degradation of oligopeptides into amino acids, which is a limiting step of rumen proteolysis [19]. Campylobacter is a microaerophilic bacterium that could consume oxygen and is involved in nitrogen metabolism [20]. In the rumen, Synergistetes is known for its capability to degrade amino acids [21]. The decreased relative abundance of Synergistetes in the rumen of goat fed a low protein diet was also reported by Zhang et al [22]. The decreased apparent digestibility of CP in the LCP group observed in our previous study [4] could be partially explained by the reduced abundances of these protein-degrading bacteria. Additionally, Prevotella have the ability to degrade fiber, partially explaining the lower acetate concentrations in the low-protein groups.

The metabolites in rumen fluid mainly contain nutrients that can be used by the host, and differences in the levels of ruminal metabolites are known to be associated with changes in microbiota [23]. In this study, most of the altered metabo- 


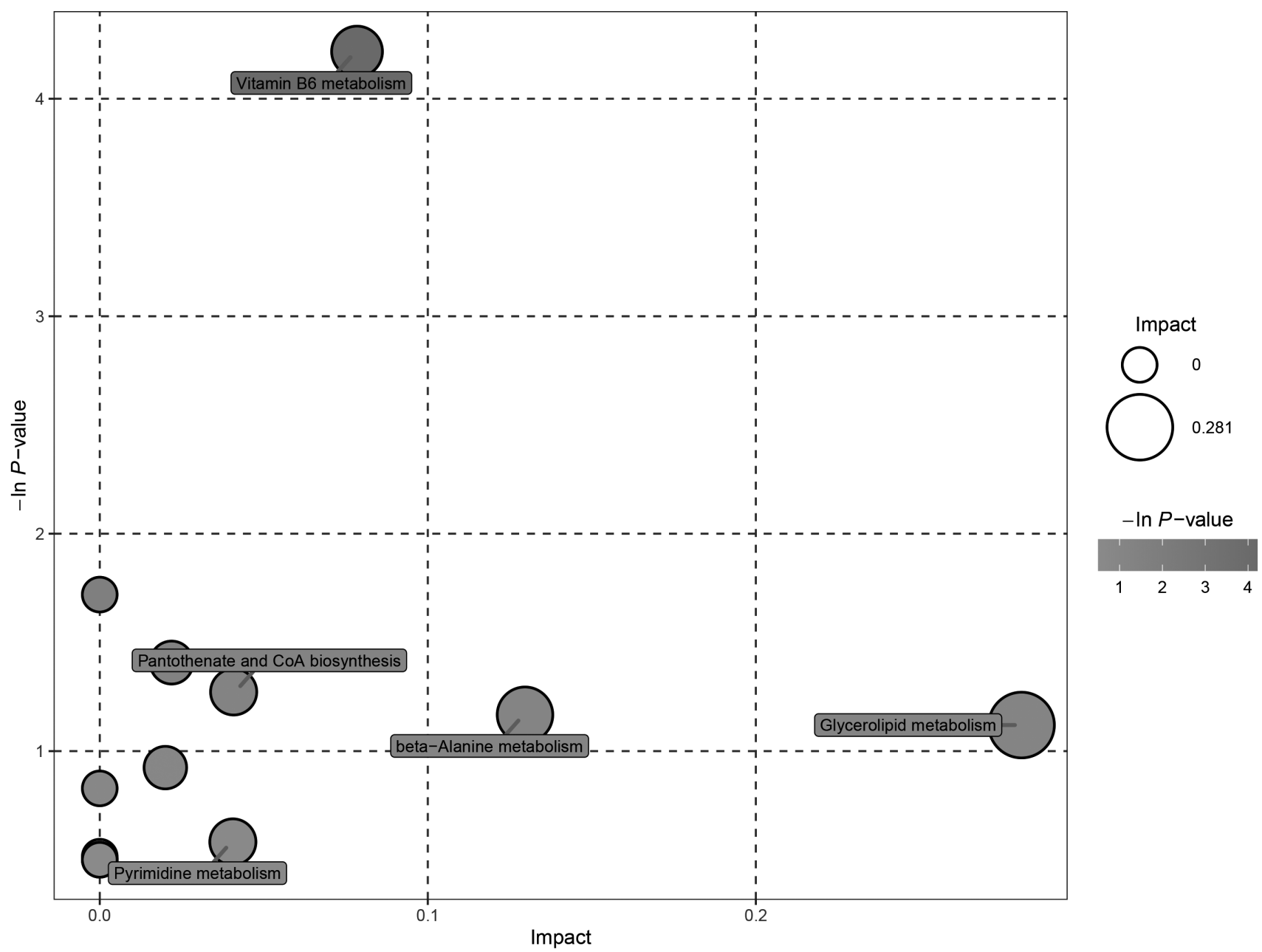

Figure 5. Ruminal metabolomics pathway analysis of goats that received the low crude protein diet compared with the normal crude protein diet. The sizes of the shapes represent the effects of low protein treatment on rumen fluid metabolism relative to the control treatments; larger shapes indicate greater effects on the pathway.

lites were decreased by low protein diet, including amino acids, peptides, and fatty acids. Amino acids and peptides can serve as precursors for the synthesis of microbial protein in the rumen, while fatty acids can provide energy for the synthesis of microbial proteins [18]. Thus, these results suggest that feeding a low protein diet to goats might decrease the microbial protein yield. The positive effect of dietary $\mathrm{CP}$ level on microbial protein synthesis is also reported by Chanthakhoun et al [24]. Glycerol was one of the significantly decreased metabolites in the LCP group in comparison with the NCP group. In the rumen, glycerol is completely fermented into VFAs, especially propionate and butyrate [25]. The decreased concentration of propionate in LCP group may be partially attributed to decreased glycerol levels.

Table 4. Rumen fluid metabolomic pathway analyses in fattening goat fed the low crude protein diet (LCP) and the normal crude protein diet (NCP)

\begin{tabular}{|c|c|c|c|c|c|}
\hline Pathway & Total & Hits $^{1)}$ & p-value ${ }^{2)}$ & Impact $^{3)}$ & Hits conpounds \\
\hline Vitamin $\mathrm{B}_{6}$ metabolism & 9 & 1 & 0.015 & 0.000 & 4-Pyridoxic acid \\
\hline Arginine and proline metabolism & 44 & 1 & 0.245 & 0.022 & Argininosuccinic acid \\
\hline Glycerolipid metabolism & 18 & 1 & 0.326 & 0.281 & Glycerol \\
\hline Alanine, aspartate and glutamate metabolism & 23 & 1 & 0.397 & 0.020 & Argininosuccinic acid \\
\hline Galactose metabolism & 26 & 1 & 0.436 & 0.000 & Glycerol \\
\hline Tryptophan metabolism & 41 & 1 & 0.596 & 0.000 & Indoleacetic acid \\
\hline
\end{tabular}

\footnotetext{
1) Hits represent the number of metabolites matches in one pathway.

2) The p-values were determined using student's t test.

${ }^{3)}$ Impact is the influencing factor of the pathway obtained by topology analysis.
} 
The pathway of vitamin $\mathrm{B}_{6}$ metabolism was significantly altered by the dietary protein content, which was characterized by decreased concentrations of pyridoxine and 4-Pyridoxic acid. Pyridoxine is one of the vitamin $B_{6}$ vitamers [26]. 4-Pyridoxic acid is the major urinary catabolite of vitamin $B_{6}$ [27]. The decreased levels of pyridoxine and 4-pyridoxic acid in the LCP group indicated downregulation of vitamin $\mathrm{B}_{6}$ metabolism. It is well known that vitamin $\mathrm{B}_{6}$, mainly synthesized in rumen, as a co-factor for more than 150 enzymes, participates in carbohydrate, protein, and lipid metabolism [28]. Meale et al [29] also reported that the efficiency of blood vitamin $\mathrm{B}_{6}$ metabolism was reduced in low feed conversion efficient bulls. Overall, the results indicating that the reduced feed efficiency in LCP group observed in our previous study might be due to the reduced efficiency of this pathway.

\section{CONCLUSION}

In conclusion, a decreased dietary protein content altered rumen microbial composition and rumen metabolism. Decreasing the protein content inhibited rumen fermentation, decreased the relative abundances of genera Prevotella, Campylobacter, Synergistetes, and TG5. Most of the metabolites that showed decreased levels in the rumen fluid in the LCP group were substrates for microbial protein synthesis, indicating a lower microbial protein yield when goats were fed LCP. The pathway enrichment analysis of the different metabolites indicated that LCP mainly affected vitamin $\mathrm{B}_{6}$ metabolism. These results enhance our understanding of ruminal bacteria and metabolites of different protein content within goat diet, and provide more informantion for the development of low protein diet.

\section{CONFLICT OF INTEREST}

We certify that there is no conflict of interest with any financial organization regarding the material discussed in the manuscript.

\section{FUNDING}

This research was funded by the National Key R\&D Program of China (No. 2018YFD0502000), Key Laboratory of Molecular Animal Nutrition of Ministry of Education of China, the Science and Technology Major Project of Anhui Province (No. 201903b06020001), National Innovative Training Program for College Student (No. 201910364046), The National Natural Science Foundation of China (No. 32002199), Anhui province Natural Science Foundation of China (No. 2008085MC86), and National Natural Science Foundation of China (No. 32002199).

\section{REFERENCES}

1. Kohn RA, Dinneen MM, Russek-Cohen E. Using blood urea nitrogen to predict nitrogen excretion and efficiency of nitrogen utilization in cattle, sheep, goats, horses, pigs, and rats. J Anim Sci 2005;83:879-89. https://doi.org/10.2527/2005. 834879x

2. Wang Y, Cao P, Wang L, Zhao Z, Chen Y, Yang Y. Bacterial community diversity associated with different levels of dietary nutrition in the rumen of sheep. Appl Microbiol Biotechnol 2017;101:3717-28. https://doi.org/10.1007/s00253-017-8144-5

3. Muscher AS, Wilkens MR, Mrochen N, Schröder B, Breves G, Huber K. Ex vivo intestinal studies on calcium and phosphate transport in growing goats fed a reduced nitrogen diet. Br J Nutr 2012;108:628-37. https://doi.org/10.1017/S00071 14511005976

4. Zhu W, Xu W, Wei CC, Zhang ZJ, Jiang CC, Chen XY. Effects of decreasing dietary crude protein level on growth performance, nutrient digestion, serum metabolites, and nitrogen utilization in growing goat kids (Capra. hircus). Animals 2020;10:151. https://doi.org/1c0.3390/ani10010151

5. Agarwal N, Kamra DN, Chaudhary LC. Rumen microbial ecosystem of domesticated ruminants. In: Puniya A, Singh R, Kamra D, editors. Rumen microbiology: From evolution to revolution, New Delhi, India: Springer Press; 2015. https:// doi.org/10.1007/978-81-322-2401-3_2

6. Zhou M, Peng YJ, Chen YH, et al. Assessment of microbiome changes after rumen transfaunation: implications on improving feed efficiency in beef cattle. Microbiome 2018;6:62. https:// doi.org/10.1186/s40168-018-0447-y

7. Hua CF, Tian J, Tian P, et al. Feeding a high concentration diet induces unhealthy alterations in the composition and metabolism of ruminal microbiota and host response in a goat model. Front Microbiol 2017;8:138. https://doi.org/10. 3389/fmicb.2017.00138

8. Clark JH, Davis CL. Some aspects of feeding high producing dairy cows. J Dairy Sci 1980;63:873-85. https://doi. org/10.3168/jds.s0022-0302(80)83021-9

9. Gao J, Sun YF, Bao Y, Zhou K, Kong DH, Zhao GY. Effects of different levels of rapeseed cake containing high glucosinolates in steer ration on rumen fermentation, nutrient digestibility and the rumen microbial community. Br J Nutr 2021;125:266-74. https://doi.org/10.1017/S000711452000 2767

10. Nicholson JK, Holmes E, Kinross J, et al. Host-gut microbiota metabolic interactions. Science 2012;336:1262-7. https://doi. org/10.1126/science. 1223813

11. National Research Council. Nutrient requirements of small ruminants, sheep, goats, cervids, and new world camelids. Washington, DC, USA: National Academy Press; 2007.

12. Hu WL, Liu JX, Ye JA, Wu YM, Guo YQ. Effect of tea saponin on rumen fermentation in vitro. Anim Feed Sci Technol 
2005;120:333-9. https://doi.org/10.1016/j.anifeedsci.2005. 02.029

13. Chaney AL, Marbach EP. Modified reagents for determination of urea and ammonia. Clin Chem 1962;8:130-2. https://doi. org/10.1093/clinchem/8.2.130

14. Wang B, Ma MP, Diao QY, Tu Y. Saponin-induced shifts in the rumen microbiome and metabolome of young cattle. Front Microbiol 2019;10:356. https://doi.org/10.3389/fmicb. 2019.00356

15. Sun HZ, Wang DM, Wang B, et al. Metabolomics of four biofluids from dairy cows: potential bimarkers for milk production and quality. J Proteome Res 2015;14:1287-98. https://doi.org/10.1021/pr501305g

16. Xia C, Rahman MAU, Yang H, et al. Effect of increased dietary crude protein levels on production performance, nitrogen utilisation, blood metabolites and ruminal fermentation of Holstein bulls. Asian-Australas J Anim Sci 2018;31: 1643-53. https://doi.org/10.5713/ajas.18.0125

17. Zhou K, Bao Y, Zhao GY. Effects of dietary crude protein and tannic acid on rumen fermentation, rumen microbiota and nutrient digestion in beef cattle. Arch Anim Nutr 2019;73: 30-43. https://doi.org/10.1080/1745039x.2018.1545502

18. National Research Council. Nutrient requirements of dairy cattle. 7th rev. ed. National Academy of Sciences: Washington, DC, USA: National Academies Press; 2001.

19. Calsamiglia S, Ferret A, Reynolds CK, Kristensen NB, van Vuuren AM. Strategies for optimizing nitrogen use by ruminants. Animal 2010;4:1184-96. https://doi.org/10.1017/ s1751731110000911

20. Mann E, Wetzels Wagner SUM, Zebeli Q, Schmitz-Esser S. Metatranscriptome sequencing reveals insights into the gene expression and functional potential of rumen wall bacteria. Front Microbiol 2018;9:43. https://doi.org/10.3389/ fmicb.2018.00043

21. Han X, Yang Y, Yan H, Wang X, Qu L, Chen Y. Rumen bacterial diversity of 80 to 110-day-old goats using $16 \mathrm{~S}$ rRNA sequencing.
PLoS One 2015;10:e0117811. https://doi.org/10.1371/journal. pone.0117811

22. Zhang XX, Li YX, Tang ZR, et al. Reducing protein content in the diet of growing goats: implications for nitrogen balance, intestinal nutrient digestion and absorption, and rumen microbiota. Animal 2020;14:2063-73. https:/doi.org/10.1017/ S1751731120000890

23. Zhu W, Su Z, Xu W, et al. Garlic skin induces shifts in the rumen microbiome and metabolome of fattening lambs. Animal 2021;15:100216. https://doi.org/10.21203/rs.3.rs57466/v1

24. Chanthakhoun V, Wanapat M, Berg J. Level of crude protein in concentrate supplements influenced rumen characteristics, microbial protein synthesis and digestibility in swamp buffaloes (bubalus bubalis). Livest Sci 2012;144:197-204. https://doi. org/10.1016/j.livsci.2011.11.011

25. Remond B, Souday E, Ouany JP. In vitro and in vivo fermentation of glycerol by rumen microbes. Anim Feed Sci Technol 1993;41:121-32. https://doi.org/10.1016/0377-8401(93)901 $18-4$

26. Garrido-Franco M. Pyridoxine 5'-phosphate synthase: de novo synthesis of vitamin B6 and beyond. Biochim Biophys Acta Proteins Proteom 2003;1647:92-7. https://doi.org/10.101 6/s1570-9639(03)00065-7

27. Gholizadeh M, Fayazi J, Asgari Y, Zali H, Kaderali L. Reconstruction and analysis of cattle metabolic networks in normal and acidosis rumen tissue. Animal 2020;10:469. https://doi. org/10.3390/ani10030469

28. Kelsay J, Baysal A, Linkswiler H. Effect of vitamin B6 depletion on the pyridoxal, pyridoxamine and pyridoxine content of the blood and urine of men. J Nutr 1968;94:490-4. https:// doi.org/10.1093/jn/94.4.490

29. Meale SJ, Morgavi DP, Cassar-Malek I, et al. Exploration of biological markers of feed efficiency in young bulls. J Agric Food Chem 2017;65:9817-27. https://doi.org/10.1021/acs. jafc.7b03503 